\title{
Prices and Efficient Assignments Without Transfers
}

\author{
Antonio Miralles and Marek Pycia*
}

December 2014

\begin{abstract}
We study the assignment of indivisible objects in environments, such as school choice, in which transfer payments are not used. Our main result shows that every efficient assignment can be decentralized through prices. We thus establish the Second Welfare Theorem for the no-transfer environments with possibly satiated agents.
\end{abstract}

\section{Introduction}

A classic insight of the Walrasian theory of markets, commonly referred to as the Second Welfare Theorem, is that every Pareto efficient assignment can be decentralized through the use of prices. ${ }^{1}$ This classic insight is predicated on the assumption that agents are locally non-satiated, an assumption that is readily satisfied in settings with money. ${ }^{2}$ However, the non-satiation assumption fails in settings without money such as the assignment of school seats in school choice programs. The assignment of scarce resources in these settings has been intensively studied recently and Pareto efficiency is a commonly accepted goal for such assignments. ${ }^{3}$

What assignments are efficient in settings without transfers? Does the insight of the Second Welfare Theorem remain valid in such settings? We address these questions in the

*Universitat Autonoma de Barcelona - Barcelona Graduate School of Economics, and University of California Los Angeles, respectively. We would like to thank Salvador Barbera, Roland Benabou, Dirk Bergemann, Simon Board, Sergiu Hart, Andrew McLennan, Jordi Masso, Moritz Meyer-ter-Vehn, Michael Richter, William Thomson, Utku Unver, William Zame, Simpson Zhang, and seminar participants at UAB and UCLA for helpful comments.

${ }^{1}$ This classic insight, also known as the Second Fundamental Theorem of Welfare Economics, was conjectured by Pareto (1909), and subsequently refined and developed by many authors, culminating in the definitive treatment by Arrow (1951) and Debreu (1951).

${ }^{2}$ Local non-satiation requires that for any agent and any assignment there is a nearby assignment that the agent strictly prefers, for instance because it leaves him with more money.

${ }^{3}$ See e.g. Abdulkadiroglu and Sonmez (2003). 
canonical no-transfer assignment model of Hylland and Zeckhauser (1979). There is a finite set of agents and objects. Each agent's utility is given by their von Neumann-Morgenstern valuations, and agents evaluate lotteries according to the expected utility theory. ${ }^{4}$ Following Hylland and Zeckhauser, we study Walrasian equilibria in which each agent is endowed with token money; the amount of token money held after the assignment has no impact on agents' utilities.

We establish the Second Welfare Theorem in this environment: despite the lack of transfers and the possibility of satiation, every efficient assignment may be supported in a Walrasian equilibrium that is decentralized via prices, just as Pareto efficient assignments in environments with transfers can be. In market design contexts, our characterization of efficient assignments allows one to restrict attention to price mechanisms at least in settings, such as large markets, where such mechanisms are incentive compatible. ${ }^{5}$ In particular, every Pareto efficient assignment may be implemented as an outcome of Hylland and Zeckhauser's mechanism with properly chosen budgets.

The problems the received approach to the Second Welfare Theorem runs into in settings with locally satiated agents are well-known (Mas-Collel, Winston, and Green, 1995), and hence it is rather surprising that the insight of the Second Welfare Theorem holds true in the canonical no-transfer environment we study. ${ }^{6}$ Indeed, whether the Second Welfare Theorem obtains in settings without transfers and with possibly satiated agents remained a puzzle except for deterministic assignments. For deterministic assignments, Abdulkadiroglu and Sonmez (1998) established a version of the Second Welfare Theorem; they showed that each deterministic assignment may be obtained via a serial dictatorship. Every serial dictatorship can be implemented via budgets and prices, but there are many efficient allocations that cannot be implemented via serial dictatorships; as we show they all can be implemented via prices. $^{7}$

\footnotetext{
${ }^{4}$ We first analyze the case where each agent demands at most one object, as in school seat assignment, and we then extend our results to the setting in which agents may demand multiple objects.

${ }^{5}$ The large market incentive compatibility of the mechanisms that set the prices endogenously has been established by Azevedo and Budish (2013). Price mechanisms are also incentive compatible in settings in which we can set prices exogenously, for instance when we have at least an approximate sense of the distribution of preferences, see Pycia (2014). He, Miralles, and Yan (2012) discuss interval incentives in two-sided markets.

${ }^{6}$ The First Welfare Theorem also obtains in our environment. It was established by Hylland and Zeckhauser (1979), and further refined by Mas-Collel (1992) and Budish, Che, Kojima, and Milgrom (2013). For instance, all equilibria are efficient if agents strictly rank any two objects. There are, of course, many environments in which the First Welfare Theorem holds true, and the Second Welfare Theorem fails, see Mas-Collel et al (1995).

${ }^{7}$ Random allocations cannot be implemented by deterministic serial dictatorships. As pointed out by Bogomolnaia and Moulin (2001), randomization over serial dictatorship is not necessarily efficient. Furthermore, notice that serial dictatorships elicit only agents' ordinal information, and as shown by Abdulkadiroglu, Che, and Yasuda (2011) mechanisms eliciting only ordinal information may lead to inefficient outcomes; this
} 
In order to prove the Second Welfare Theorem we develop a new approach to the proof because the failure of local non-satiation implies that the Separating Hyperplane Theorem commonly used to prove the Second Welfare Theorem guarantees only the existence of a separating hyperplane that may have non-empty intersections with the set of Pareto-dominant aggregate assignments. ${ }^{8}$ Facing the resulting prices, some agents might afford to buy bundles they strictly prefer over their assignment; this situation is called a quasi-equilibrium. In contrast, we prove the existence of a separating hyperplane that is disjoint with the set of Pareto-dominant aggregate assignments. Facing the resulting prices, no agent can afford a bundle they would prefer over their assignment, and the prices support the assignment as an equilibrium. We provide two proofs of this result: a direct proof and a proof that builds on McLennan's (2002) Separating Hyperplane Theorem. ${ }^{9}$

Prior work on no-transfer assignments related price mechanisms to efficiency but only in conjunction with other strong requirements. In continuum economies, Thomson and Zhou (1993) related efficient, symmetric, and consistent mechanisms to Hylland and Zeckhauser's mechanism, and Ashlagi and Shi (2014) showed that any efficient, symmetric, and strategyproof random assignment can be expressed as the result of the Hylland and Zeckhauser mechanism. ${ }^{10}$ In contrast, we do not rely on symmetry, consistency, or strategy-proofness, and we prove our results for all finite economies. ${ }^{11}$

Finally, we contribute to the literature on the Second Welfare Theorem beyond the standard exchange economy model. Anderson (1988) proved the Second Welfare Theorem for exchange economies with nonconvex preferences; in contrast with us, he maintained the assumption of local non-satiation. Richter and Rubinstein (2014) propose a general convex geometry approach to welfare economics based on the concept of "primitive equilibrium," where a strict linear ordering arranges alternatives in order to create "budget" sets. They prove a Second Welfare Theorem for the primitive equilibrium concept; when preferences

inefficiency does not necessarily vanish as the market becomes large, as shown by Pycia (2014).

${ }^{8}$ While the full separation obtains if one of the separated sets is open, this assumption fails in our setting. Section 3 provides an example illustrating the failure of openness, and a more detailed discussion of why the standard techniques do not work.

${ }^{9}$ We would like to thank Andrew McLennan for suggesting the second of these two approaches. The advantage of the proof based on McLennan's Separating Hyperplane Theorem is that it provides more information about the structure of the problem while the direct approach establishes the theorem with minimal conceptual structure. For prior work employing McLennan's theorem, see Manea (2008) and Carroll (2010).

${ }^{10}$ Makowski, Ostroy, and Segal (1999) showed a similar result for the classical exchange economies.

${ }^{11}$ Related to our work are also other papers relying on the idea of using token money to allocate objects in the absence of transfers. Token money mechanisms have been extended beyond the canonical Hylland and Zekchauser setting by, for instance, Sonmez and Unver (2010), Budish (2013), Manjunath (2014), and Miralles (2014). Hafalir and Miralles (2014) analyze the utilitarian efficiency of such market approaches. None of these papers establishes a Second Welfare Theorem. 
are strictly monotone, their primitive equilibrium concept corresponds to the standard equilibrium concept, however, when specialized to our setting, this equilibrium concept becomes equivalent to the quasi-equilibrium discussed above. ${ }^{12}$

\section{Model}

We study a finite economy with agents $i, j \in I=\{1, \ldots,|I|\}$ and indivisible objects $x, y \in$ $X=\{1, \ldots,|X|\}$. Each object $x$ is represented by a number of identical copies $|x| \in \mathbb{N}$. By $S=(|x|)_{x \in X}$ we denote the total supply of object copies in the economy. If agents have outside options, we treat them as objects in $X$; in particular, this implies that $\sum_{x \in X}|x| \geq|I|$.

We assume initially that agents demand at most one copy of an object; we relax this assumption in Section 5. We allow random assignments and denote by $q_{i}^{x} \in[0,1]$ the probability that agent $i$ obtains a copy of object $x$. Agent $i$ 's random assignment $q_{i}=$ $\left(q_{i}^{1}, \ldots, q_{i}^{|X|}\right)$ is a probability distribution. The economy-wide assignment $\left(q_{i}^{x}\right)_{i \in I, x \in X}$ is feasible if $\sum_{i \in I} q_{i}^{x} \leq|x|$. Let $\mathcal{A}$ denote the set of economy-wide random assignments, and $\mathcal{F} \subset \mathcal{A}$ denote the set of feasible random assignments. We call an assignment pure, or deterministic, if each of its elements $q_{i}^{x}$ is either 0 or 1 . By the Birkhoff-von Neumann theorem, a feasible random assignment can be expressed as a lottery over feasible pure assignments.

Agents are expected utility maximizers, and agent $i$ 's utility from random assignment $q_{i}$ equals the scalar product $u_{i}\left(q_{i}\right)=v_{i} \cdot q_{i}$ where $v_{i}=\left(v_{i}^{x}\right)_{x \in X} \in[0, \infty)^{|X|}$ is the vector of agent $i$ 's von Neumann-Morgenstein valuations for objects $x \in X$.

We study the connection between two concepts: efficiency and equilibrium. A feasible random assignment $Q^{*} \in \mathcal{F}$ is Pareto efficient (or, simply, efficient) if no other feasible random assignment $Q \in \mathcal{F}$ is weakly preferred by all agents and strictly preferred by some agents.

A random assignment $Q^{*}$ and a price vector $p^{*}$ constitute an equilibrium for a budget vector $w^{*} \in \mathbb{R}_{+}^{|I|}$ if $Q^{*}$ is feasible, $p^{*} \cdot q_{i}^{*} \leq w_{i}^{*}$ for any $i \in I$, and $u_{i}\left(q_{i}\right)>u_{i}\left(q_{i}^{*}\right) \Longrightarrow p^{*} \cdot q_{i}>w_{i}^{*}$ for any $\left(q_{i}\right)_{i \in I} \in \mathcal{A}$.

\footnotetext{
${ }^{12}$ In Section 3 we provide an example of a quasi-equilibrium which is not an equilibrium; this quasiequilibrium is a primitive equilibrium in the sense of Richter and Rubinstein. To the best of our knowledge the above discussion covers all extensions of the Second Welfare Theorem beyond the standard strictly monotone and convex setting. Of course, the literature on Walrasian equilibria beyond this setting is richer, and - in addition to the papers cited above (including in footnotes) —includes, for instance, Bergstrom (1976), Manelli (1991), and Hara (2005) who focused on equilibrium existence and core convergence rather than on the Second Welfare Theorem.
} 


\section{Main Result: The Second Welfare Theorem}

Our main result is the counterpart of the second welfare theorem for the setting without transfers.

Theorem 1. (The Second Welfare Theorem) If $Q^{*} \in \mathcal{F}$ is Pareto-efficient, then there is a vector of budgets $w^{*} \in \mathbb{R}_{+}^{|I|}$ and a vector of prices $p^{*} \in \mathbb{R}_{+}^{|X|}$ such that $Q^{*}$ and $p^{*}$ constitute an equilibrium with budgets $w^{*}$.

Before laying out the proof, let us compare our problem to the standard second welfare theorem with transfers and preferences that are convex and strictly monotonic. The well-known argument in the standard setting relies on the celebrated separating hyperplane theorem and it goes as follows. The set of aggregate feasible assignments is convex, and, given an efficient assignment $Q^{*}=\left(q_{i}^{*}\right)_{i \in I}$ we want to implement, the set of (infeasible) aggregate assignments that Pareto dominate $Q^{*}$ is convex as well. Since these two sets are disjoint, the separating hyperplane theorem tells us there exists a hyperplane that separates them. The normal vector to this hyperplane gives us a price vector $p^{*}$, and the separation means that

$$
u_{i}\left(q_{i}\right)>u_{i}\left(q_{i}^{*}\right) \Longrightarrow p^{*} \cdot q_{i} \geq w_{i}^{*}
$$

for any $i \in I$ and for any $\left(q_{i}\right)_{i \in I} \in \mathcal{A}$. In other words, the separating hyperplane theorem allows us to find a price vector $p^{*}$ that implements the efficient assignment $Q^{*}$ as a so-called quasi-equilibrium.

The final step of the standard proof is then to show that this quasi-equilibrium is in fact an equilibrium, that is

$$
u_{i}\left(q_{i}\right)>u_{i}\left(q_{i}^{*}\right) \Longrightarrow p^{*} \cdot q_{i}>w_{i}^{*}
$$

for any $i \in I$ and for any $\left(q_{i}\right)_{i \in I} \in \mathcal{A}$. This last step is by contradiction: we take an assignment $Q=\left(q_{i}\right)_{i \in I}$ that Pareto dominates $Q^{*}$ while costing the same as $Q^{*}$; in the neighborhood of $Q$ we then find an assignment that still Pareto dominates $Q^{*}$ while being cheaper than it. This is a contradiction as in quasi-equilibrium no cheaper assignment can Pareto dominate $Q^{*}$.

It is this final step of the standard proof that fails in our setting. The standard separating hyperplane theorem separates the Pareto dominating assignments from the feasible ones, in the sense of inequality (1), but it does not fully separate the two sets, in the sense of inequality (2). Unlike in the standard setting, in the setting with locally satiated preferences and without transfers, not every quasi-equilibrium is an equilibrium. The reason is as follows. In the standard setting with strongly monotone preferences no good can have a price of zero 


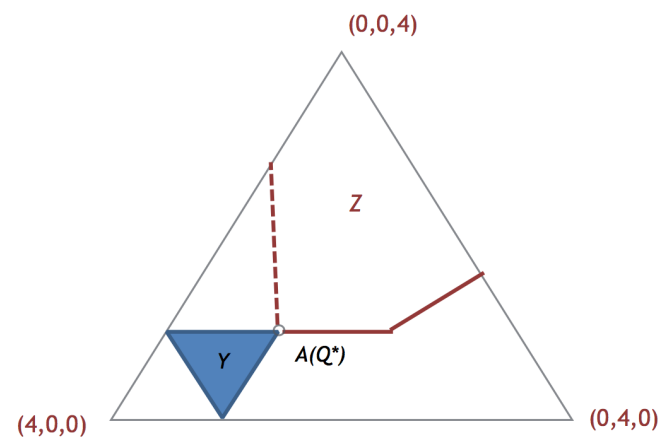

Figure 1: The simplex of "full-consumption" aggregate assignments. Assignment $A\left(Q^{*}\right)$ is on the intersection of the boundaries of sets $Y$ and $Z$.

since agents would demand an infinite amount of such a good. In contrast, zero prices are the staple of our setting as recognized already by Hylland and Zeckhauser (1979). In particular, in a quasi-equilibrium an agent may be assigned a zero-price object while he strictly prefers another zero-price object. As an illustration consider the following example.

Example 1. Consider an economy with four agents and three objects. Two of the agents have von Neumann-Morgenstern utility vector $v=\left(\frac{1}{2}, 0,1\right)$, and the remaining two agents have the utility vector $v^{\prime}=\left(0,1, \frac{1}{2}\right)$. Suppose that there are three copies of object 1 , one copy of object 2 , and one copy of object 3 . The following allocation $Q^{*}$ is then Pareto-efficient: $v$-agents obtain $q^{*}=\left(\frac{1}{2}, 0, \frac{1}{2}\right)$ and $v^{\prime}$-agents obtain $q^{* \prime}=\left(\frac{1}{2}, \frac{1}{2}, 0\right)$.

The resulting aggregate assignment $A\left(Q^{*}\right)$ is $(2,1,1)$. Figure 1 places this point in the barycentric simplex of aggregate assignments in which exactly four units are assigned, that is such that for each agent the sum of probabilities of the three goods is 1 (the full-consumption simplex). Set $Y$ represents feasible aggregate assignments in the simplex; it is the triangle spanned by $(2,1,1),(3,0,1)$ and $(3,1,0)$. Set $Z$ represents all aggregate assignments $A(Q)$ in the simplex such that there exists an assignment $Q$ in which all agents are weakly betteroff than under $Q^{*}$ and at least one agent is strictly better-off, and such that $A(Q)$ is the aggregate assignment of $Q$ (these assignments are, of course, not feasible). Set $Z$ has five corners:

- $(2,1,1)$, the aggregate assignment corresponding to $Q^{*}$,

- $(1,2,1)$, the aggregate assignment when $v$-agents obtain $q^{*}$ and $v^{\prime}$-agents obtain $(0,1,0)$,

- $\left(0,2 \frac{1}{2}, 1 \frac{1}{2}\right)$, the aggregate assignment when $v$-agents obtain $\left(0, \frac{1}{4}, \frac{3}{4}\right)$ and $v^{\prime}$-agents obtain $(0,1,0)$,

- $(0,0,4)$, the aggregate assignment when each agent obtains good 3 
- $(1,0,3)$, the aggregate assignment when $v$-agents obtain $q^{*}$ and $v^{\prime}$-agents obtain $(0,0,1)$.

Only the middle three corners belong to $Z$, and one of the borders of $Z$, the dashed line, is disjoint with $Z$. In particular, the set $Z$ is neither open nor closed.

Restricting attention to the assignments in the simplex, there is a horizontal hyperplane separating $Y$ and $Z$. This hyperplane corresponds to prices $p^{3}>p^{2}=p^{1}=0$. When $v$-agents have budget $\frac{1}{2} p^{3}$ and $v^{\prime}$-agents have budget zero, these prices support $Q^{*}$ as a quasi-equilibrium but not as an equilibrium. Indeed, $v^{\prime}$-agents would rather buy a sure copy of object 2 than the lottery $q^{* \prime}$, and both these outcomes have the price of zero.

We develop a new proof approach to establish the second welfare theorem and to address the difficulties illustrated in Example 1. To understand our approach observe that in Example 1, there are non-horizontal hyperplanes that fully separate $Y$ and $Z$ (in the full-consumption simplex). We show that this is always the case. A key step in the proof is the following new Separating Hyperplane Theorem that establishes that under conditions that - as we will shortly see - are always satisfied in the no-transfer assignment problem, full separation is possible.

Lemma 1. (Full Separation Lemma) Suppose $Y \subset \mathbb{R}^{n}$ is a closed and convex polytope, suppose $Z \subset \mathbb{R}^{n}$ is convex and non-empty, and $\bar{Z} \subset \mathbb{R}^{n}$ is a closed and convex polytope containing $Z$. Suppose further that $Z \cap Y=\emptyset$ and that for all $y \in Y \cap \bar{Z}, \delta \in \mathbb{R}^{n}$, and $\varepsilon>0$ if $y+\delta \in Z$, then $y-\varepsilon \delta \notin \bar{Z}$. Then, there exists a price vector $p \in \mathbb{R}_{+}^{n}$ and a budget $w \in \mathbb{R}$ such that for any $z \in Z$ and $y \in Y$ we have $p \cdot z>w \geq p \cdot y$ and such that for any $\bar{z} \in \bar{Z}$ and $y \in Y$ we have $p \cdot \bar{z} \geq w \geq p \cdot y$.

We provide the proof of the lemma in the appendix.

We can easily visualize the statement of the lemma in the context of Example 1. Both the set $Y$ of feasible aggregate assignments and the set $Z$ of (infeasible) aggregate assignments that Pareto dominate $Q^{*}$ are polytopes. Our separation lemma says that if every line through $Q^{*}$ and a point in $Z$ has points that belong to the closure of $Z$ only on one side of $Q^{*}$, then there exists a fully separating hyperplane. The line assumption is satisfied in our example.

The rest of the proof of the second welfare theorem revolves around showing that indeed the assumption of the lemma is satisfied: no line through $Q^{*}$ can intersect the closure of $Z$ on both sides of $Q^{*}$ (see the highlighted claim in the proof below).

Proof of the Second Welfare Theorem. For any random assignment $Q \in \mathcal{A}$, we define the aggregate assignment $A(Q)$ associated with $Q$ to be $\sum_{i \in I} q_{i}$, and we write $Q \succ Q^{*}$ when $u_{i}\left(q_{i}\right) \geq u_{i}\left(q_{i}^{*}\right)$ for every $i \in I$ with at least one strict inequality. 
We can assume that no agent $i$ obtains their most-preferred object. Indeed, suppose each agent in set $J \subseteq I$ obtains his or her most preferred object, and that $\left.Q^{*}\right|_{I-J}$ and $p^{*}$ are an equilibrium for some profile of budgets. Then, $Q^{*}$ and $p^{*}$ are an equilibrium for the same budgets of agents in $I-J$ and any sufficiently high budgets of agents in $J$.

Let $Z=\left\{A(Q): Q \succ Q^{*}, Q \in \mathcal{A}\right\}$, and notice that the above assumption implies that $Z$ is non-empty. Furthermore, $Z$ is convex. Let $\bar{Z}=\mathrm{Cl}(Z)$ be the topological closure of $Z$, and notice that $\bar{Z}$ is a non-empty convex polytope. Let $Y=\{A(Q): Q \in \mathcal{F}\}$ be the set of aggregate feasible random assignments. This set is a closed and convex polytope, and the efficiency of $Q^{*}$ implies that $Z \cap Y=\varnothing$.

To use the full separation lemma, we need the following

Claim. For any $y \in Y \cap \bar{Z}, \delta \in \mathbb{R}^{|X|}$ and $\varepsilon>0$, if $y+\delta \in Z$ then $y-\varepsilon \delta \notin \bar{Z}$.

Proof of the claim: If $y+\delta \in Z$ then there is a $Q \succ Q^{*}$ such that $A(Q)=y+\delta$. By way of contradiction, assume $y-\varepsilon \delta \in \mathrm{Cl}(Z)$. Thus, there is a $\tilde{Q}=\left(\tilde{q}_{i}\right)_{i \in I}$ such that $u_{i}\left(\tilde{q}_{i}\right) \geq u_{i}\left(q_{i}^{*}\right)$ for every $i \in I$ and $A(\tilde{Q})=y-\varepsilon \delta$. Then, the random assignment $\bar{Q}=\frac{\varepsilon}{1+\varepsilon} Q+\frac{1}{1+\varepsilon} \tilde{Q}$ is feasible, and the choice of $Q$ and $\tilde{Q}$ and the linearity of utility $u_{i}(\cdot)$ in probabilities imply that $\bar{Q} \succ Q^{*}$. But this contradicts the fact that $Q^{*}$ is ex-ante Pareto-efficient, proving the claim.

This claim and the full separation lemma imply that there exists a price vector $p \in \mathbb{R}_{+}^{|X|}$ and a budget $w \in \mathbb{R}$ such that $p \cdot z>w \geq p \cdot y$, for any $z \in Z$ and $y \in Y$. Since $Q^{*}$ is feasible $\sum_{i \in I} q_{i}^{*} \in Y$ and thus $p \cdot \sum_{i \in I} q_{i}^{*} \leq w$. Furthermore, $p \cdot \sum_{i \in I} q_{i}^{*} \geq w$ because $Q^{*} \in \mathrm{Cl}(Z)$. We conclude $p \cdot \sum_{i \in I} q_{i}^{*}=w$. Now, if we take some $q_{i}$ that some agent $i \in I$ strictly prefers to $q_{i}^{*}$, then $q_{i}+\sum_{j \in I \backslash\{i\}} q_{j}^{*} \in Z$, and we have $p \cdot\left(q_{i}+\sum_{j \in I \backslash\{i\}} q_{j}^{*}\right)>w=p \cdot\left(q_{i}^{*}+\sum_{j \in I \backslash\{i\}} q_{j}^{*}\right)$. Consequently we have $p \cdot q_{i}>p \cdot q_{i}^{*}$, proving that $p$ and $Q^{*}$ constitute an equilibrium for budgets $w_{i}^{*}=p \cdot q_{i}^{*}$. QED

In Appendix B, we provide an alternative proof of our main result that is based on McLennan's (2002) Separating Hyperplane Theorem.

\section{Multiple-Unit Demand}

Let us extend the results to the multiple-unit demand setting studied in Budish et al (2013). As before we have a set of agents $I$ and a set of objects $X$. As before, each object $x \in X$ has a finite number of copies $|x|$. Each agent $i$ is endowed with von Neumann-Morgenstern valuations $v_{i}=\left(v_{i}^{1}, \ldots, v_{i}^{|X|}\right) \in[0,+\infty)^{|X|}$ and with the set of consumption-feasible bundles, $X_{i} \subset \mathbb{R}^{X}$. The utility from bundle $q_{i}=\left(q_{i}^{1}, \ldots, q_{i}^{|X|}\right) \in X_{i}$ is the scalar product $q_{i} v$; the 
utility from other bundles is zero. For simplicity, we focus on sets $X_{i}$ such that $q_{i} \in X_{i}$ if the total quantity consumed $\left|q_{i}\right|=q_{i}^{1}+\ldots+q_{i}^{|X|}$ is less than a quota $|i|$ and the agent consumes at most quantity 1 of each object. ${ }^{13}$ The definitions of efficiency and pseudomarket equilibrium translate word-for-word to this multiple-unit demand setting. Budish et al. (2013) showed that any random assignment $\left(q_{i}\right)_{i \in I}$ that satisfies the above constraints can be implemented as a lottery over deterministic assignments. They also proved the First Welfare Theorem for the case of equal budgets.

The Second Welfare Theorem (Theorem 1) continues to hold true, and its proof follows the same steps as in the single-unit demand case.

Theorem 2. (The Second Welfare Theorem with Multi-Unit Demands) If $Q^{*} \in \mathcal{F}$ is ex-ante Pareto-efficient, then there is a vector of budgets $w^{*} \in \mathbb{R}_{+}^{|I|}$ and a vector of prices $p^{*} \in \mathbb{R}_{+}^{|X|}$ such that $Q^{*}$ and $p^{*}$ constitute a pseudomarket equilibrium with budgets $w^{*}$.

\section{Conclusion}

We have established the second welfare theorem for the assignment problem without transfers. Thus, in this setting every efficient assignment can be accomplished by a price mechanism. In addition to the substantive message, we developed a new approach to analyzing markets in which agents' preferences are only weakly convex and fail the non-satiation assumption.

\section{A Proof of The Full Separation Lemma}

We say that $X$ is fully separated from $Y$ when $p \cdot x>w \geq p \cdot y$ and we say that $Z$ is separated from $Y$ when $p \cdot z \geq w \geq p \cdot y$.

The lemma is easy when $n=1$. To prove the general case, we will proceed by induction supposing that the lemma is true in dimensions lower than $n \geq 2$.

First notice that $Y \cap \bar{Z}$ is of dimension lower than $n$. Indeed, if $Y \cap \bar{Z}$ is of dimension $n$ then there would be an open ball $B \subset Y \cap \bar{Z}$ around point $y^{*} \in Y \cap \bar{Z}$. Taking any $z \in Z$ and setting $\delta=z-y^{*}$ we would find an $\epsilon>0$ and a point $y-\epsilon \delta \in B$ contrary to $y-\epsilon \delta \notin \bar{Z}$.

\footnotetext{
${ }^{13}$ The single-unit demand setting is the special case of the multi-unit demand setting, in which $|i|=1$ for each agent $i$. Furthermore, Theorems 1 and 2 remain true for any type of consumption constraints $X_{i}$ that satisfies Budish et al's hierarchy condition. Nguyen et al (2014) discuss the implementation problems with general non-linear specifications, while Budish et al show how to use Milgrom's (2009) integer assignment messages to reduce certain non-linear preferences to the linear setting we study.
} 
Since, $Y \cap \bar{Z}$ is of dimension lower than $n$, the separating hyperplane theorem implies that there is a hyperplane $H$ that separates $Y$ and $\bar{Z}$. If $Z$ is disjoint with $H$ or if $Y$ is disjoint with $H$ then the lemma is true. Suppose thus that $H \cap Z$ and $H \cap Y$ are non-empty.

Notice that $Z^{\prime}=Z \cap H, Y^{\prime}=Y \cap H$, and $\bar{Z}^{\prime}=\bar{Z} \cap H$ satisfy the assumptions of the lemma in the linear space $H$. By the inductive assumption, there is an $n-2$ dimensional hyperplane $H^{\prime} \subsetneq H$ fully separating $Z^{\prime}$ from $Y^{\prime}$ in $H$, and separating $\bar{Z}^{\prime}$ from $Y^{\prime}$ in $H$. Notice that $H^{\prime}$ splits $H$ into two open half-spaces. Let $H^{\bar{Z}} \subset H$ be the open half-space with empty intersection with $Y^{\prime}$ (and hence with $Y$ ) and $H^{Y} \subset H$ be the open half-space with empty intersection with $\bar{Z}^{\prime}$ (and hence with $\bar{Z}$ ).

To conclude the proof, look at $n-1$ dimensional hyperplanes that contain $H^{\prime}$. Since $\bar{Z}$ and $Y$ are polytopes, at least one of these hyperplanes, say $H^{2} \neq H$, also separates $\bar{Z}$ and $Y$. Indeed, if none of them did then but $H$ then either $H^{Y}$ would need to have a nonempty intersection with $\bar{Z}$, or $H^{\bar{Z}}$ would need to have a non-empty intersection with $Y$, a contradiction.

Now, both $H$ and $H^{2}$ separate the $n$-dimensional space into two open half-spaces. Let $H(\bar{Z})$ be the half-space bounded by $H$ and disjoint with $Y$, and define analogously $H^{2}(\bar{Z})$, $H(Y)$, and $H^{2}(Y)$. Now $H(\bar{Z}) \cap H^{2}(Y)$ is disjoint with both $Y$ and $\bar{Z}$ and $\left(H(Y) \cap H^{2}(\bar{Z})\right) \cup$ $\left(H(\bar{Z}) \cap H^{2}(Y)\right)$ contain many hyperplanes. Take any such hyperplane $H^{*}$. This hyperplane separates $\bar{Z}$ from $Y$ and it fully separates $Z$ from $Y$. QED ${ }^{14}$

\section{B An Alternative Proof of the Second Welfare Theo- rem}

We provide an alternative proof of the Second Welfare Theorem based on McLennan's (2002) Separating Hyperplane Theorem. Let $P$ be a polyhedron in $\mathbb{R}^{n} .^{15}$ A proper face of $P$ is an intersection of $P$ and a hyperplane $H$ that splits $\mathbb{R}^{n}$ into two half spaces $H^{+}$and $H^{-}$ where $P \subset H^{+}{ }^{16}$ The affine hull of a set, denoted aff, is the collection of all finite linear combinations of points in the set with weights adding up to 1 (with negative weights allowed, as opposed to a convex hull). A face of $P$ is a set that is either a proper face of $P$ or $P$ itself.

Lemma 2. (McLennan's Separating Hyperplane Theorem) Suppose $Y \subset \mathbb{R}^{n}$ and $\bar{Z} \subset \mathbb{R}^{n}$ are polyhedra. Let $F_{Y}$ and $F_{\bar{Z}}$ be the smallest faces of respectively $Y$ and $\bar{Z}$ that

\footnotetext{
${ }^{14}$ An alternative proof of Lemma 1 can be based on McLennan (2002) Separating Hyperplane Theorem; we would like to thank Andrew McLennan for making this point. McLennan's theorem can also be used to prove our main result; we provide such an alternative proof of our main result in Appendix B.

${ }^{15} \mathrm{~A}$ polyhedron is the intersection of a finite number of half spaces defined by corresponding hyperplanes.

${ }^{16}$ According to this definition, vertices and edges are also considered as proper faces. The empty set is also a proper face of $P$ as long as $P \neq \mathbb{R}^{n}$.
} 
contain $Y \cap \bar{Z}$, and assume that aff $\left(F_{Y} \cup F_{\bar{Z}}\right) \neq \mathbb{R}^{n}$. Then there is a hyperplane $H$ that separates $\mathbb{R}^{n}$ into two half spaces $H^{+}$and $H^{-}$where $Y \subset H^{-}$and $\bar{Z} \subset H^{+}$such that $Y \cap H=F_{Y}$ and $\bar{Z} \cap H=F_{\bar{Z}}$.

Alternative Proof of the Second Welfare Theorem. For any random assignment $Q \in \mathcal{A}$, we define the aggregate assignment $A(Q)$ associated with $Q$ to be $\sum_{i \in I} q_{i}$. We write $Q \succ Q^{*}$ when $u_{i}\left(q_{i}\right) \geq u_{i}\left(q_{i}^{*}\right)$ for every $i \in I$ with at least one strict inequality. We write $Q \succeq Q^{*}$ when $u_{i}\left(q_{i}\right) \geq u_{i}\left(q_{i}^{*}\right)$ for every $i \in I$.

We can assume that no agent $i$ obtains their most-preferred object. Indeed, suppose each agent in set $J \subseteq I$ obtains his or her most preferred object, and that $\left.Q^{*}\right|_{I-J}$ and $p^{*}$ are an equilibrium for some profile of budgets. Then, $Q^{*}$ and $p^{*}$ are an equilibrium for the same budgets of agents in $I-J$ and any sufficiently high budgets of agents in $J$.

Let $Z=\left\{A(Q): Q \succ Q^{*}, Q \in \mathcal{A}\right\}$, and notice that the above assumption implies that $Z$ is non-empty and convex. Let $\bar{Z}=\mathrm{Cl}(Z)$ be the topological closure of $Z$, and notice that $\bar{Z}$ is a non-empty polyhedron in the $n-1$ dimensional subspace $S=\left\{a \in \mathbb{R}^{|X|}: \sum_{x=1}^{|X|} a^{x}=|I|\right\}$. Let $Y=\{A(Q): Q \in \mathcal{F}\}$ be the set of aggregate feasible random assignments. This set is also a polyhedron in the same subspace $S$. The efficiency of $Q^{*}$ implies that $Z \cap Y=\varnothing$. Let $F_{Y}$ and $F_{\bar{Z}}$ be the smallest faces of respectively $Y$ and $\bar{Z}$ that contain $Y \cap \bar{Z}$. Defining the relative interior (denoted ri) of a set as the interior of this set in the linear space defined by the affine hull of this set, we conclude that the relative interiors of $Y$ and $\bar{Z}$ have an empty intersection. Thus, aff $\left(F_{Y} \cup F_{\bar{Z}}\right) \varsubsetneqq S \varsubsetneqq \mathbb{R}^{|X|}$. According to McLennan's Separating Hyperplane Theorem, there is a hyperplane $H$ separating $Y$ from $\bar{Z}$ and such that $H \cap \bar{Z}=$ $F_{\bar{Z}}$. To prove the full separation of $Z$ and $Y$, it is thus sufficient to show that $Z \cap F_{\bar{Z}}=\varnothing$.

Claim. $Z \cap F_{\bar{Z}}=\varnothing$

Proof of the claim: We omit simple cases in which $F_{\bar{Z}}$ is either empty or a singleton. In the latter case $F_{\bar{Z}}=\left\{A\left(Q^{*}\right)\right\}$ and it is clear that $Z \cap F_{\bar{Z}}=\varnothing$. So we consider remaining cases. By way of contradiction, let $A(Q) \in Z \cap F_{\bar{Z}}$ where $Q \succ Q^{*}$.

It must be the case that $Y \cap \bar{Z}$ has a nonempty intersection with the relative interior of $F_{\bar{Z}}$, that is $Y \cap \operatorname{ri}\left(F_{\bar{Z}}\right) \neq \varnothing$. Otherwise $F_{\bar{Z}}$ would not be the smallest face of $\bar{Z}$ containing $Y \cap \bar{Z}$ because if the intersection of these two polyhedra has no points in the relative interior of $F_{\bar{Z}}$, then this intersection must have all of its points in one face of $F_{\bar{Z}}$ that is not $F_{\bar{Z}}$ itself.

Select any $a \in Y \cap \operatorname{ri}\left(F_{\bar{Z}}\right)$. Since $A(Q) \in F_{\bar{Z}}$, for $\varepsilon>0$ small enough, we have $a-$ $\varepsilon[A(Q)-a] \in F_{\bar{Z}}$. There exists $\tilde{Q} \succeq Q^{*}$ such that $A(\tilde{Q})=a-\varepsilon[A(Q)-a]$ because $\left.F_{\bar{Z}} \subset \bar{Z}=\operatorname{Cl}(Z)=\left\{z: \exists \tilde{Q} \in \mathcal{A} \mid A(\tilde{Q})=z, \tilde{Q} \succeq Q^{*}\right\}\right)$. Let us define the random assignment $\bar{Q}=\frac{\varepsilon}{1+\varepsilon} Q+\frac{1}{1+\varepsilon} \tilde{Q}$. Since $A(\bar{Q})=a$ this assignment is feasible, $\bar{Q} \in Y$. The choice of $Q$ and 
$\tilde{Q}$ and the linearity of utility $u_{i}(\cdot)$ in probabilities imply that $\bar{Q} \succ Q^{*}$. But this contradicts the fact that $Q^{*}$ is ex-ante Pareto-efficient, proving the claim.

This claim and McLennan's Separating Hyperplane Theorem imply that there exists a price vector $p \in \mathbb{R}^{|X|}$ and a budget $w \in \mathbb{R}$ such that $p \cdot z>w \geq p \cdot y$, for any $z \in Z$ and $y \in Y$. (For this we simply define $H=\left\{z \in \mathbb{R}^{n}: p \cdot z=w\right\}$ ). Since both $Z$ and $Y$ contain only vectors whose elements add up to the same number $|I|$, for any $\alpha>0, \beta \in \mathbb{R}$ we have that $H_{\alpha \beta}=\left\{z \in \mathbb{R}^{n}:[\alpha p+(\beta, \ldots, \beta)] \cdot z=w\right\}$ also fully separates $Z$ from $Y$. Notice that without loss of generality we can take $p \in \mathbb{R}_{+}^{|X|}$ and $w \in \mathbb{R}_{+}$.

Since $Q^{*}$ is feasible $\sum_{i \in I} q_{i}^{*} \in Y$ and thus $p \cdot \sum_{i \in I} q_{i}^{*} \leq w$. Furthermore, $p \cdot \sum_{i \in I} q_{i}^{*} \geq w$ because $A\left(Q^{*}\right) \in \mathrm{Cl}(Z)$. We conclude $p \cdot \sum_{i \in I} q_{i}^{*}=w$. Now, if we take some $q_{i}$ that some agent $i \in I$ strictly prefers to $q_{i}^{*}$, then $q_{i}+\sum_{j \in I \backslash\{i\}} q_{j}^{*} \in Z$, and we have $p \cdot\left(q_{i}+\sum_{j \in I \backslash\{i\}} q_{j}^{*}\right)>$ $w=p \cdot\left(q_{i}^{*}+\sum_{j \in I \backslash\{i\}} q_{j}^{*}\right)$. Consequently we have $p \cdot q_{i}>p \cdot q_{i}^{*}$, proving that $p$ and $Q^{*}$ constitute an equilibrium for budgets $w_{i}^{*}=p \cdot q_{i}^{*}$. QED

\section{References}

[1] Arrow K. (1951) "An Extension of the Basic Theorems of Classical Welfare Economics." In: Proceedings of the Second Berkeley Symposium on Mathematical Statistics and Probability. University of California Press.

[2] Abdulkadiroglu, Atila, Che Yeon-Koo, and Yasuke Yasuda. (2011) "Resolving Conflicting Preferences in School Choice: the Boston Mechanism Reconsidered," American Economic Review 101(1), 399-410.

[3] Abdulkadirglu, Atila and Tayfun Sonmez. (1998) "Random Serial Dictatorship and the Core from Random Endowments in House Allocation Problems." Econometrica 66, 689701.

[4] Abdulkadirglu, Atila and Tayfun Sonmez. (2003) "School Choice: A Mechanism Design Approach." American Economic Review 93(3), 729-747.

[5] Anderson, Robert M. (1988) "The Second Welfare Theorem with Nonconvex Preferences." Econometrica 56(2), 361-382.

[6] Ashlagi I. and Shi P. (2014) "Optimal Allocation Without Money: an Engineering Approach", forthcoming at Management Science.

[7] Azevedo E. and Budish. E. (2013) Strategyproofness in the Large, working paper. 
[8] Bergstrom, Ted C. (1976) "How to Discard 'Free Disposability' —at No Cost," Journal of Mathematical Economics, 3, 131-134.

[9] Bogomolnaia, A. and H. Moulin (2001) "A New Solution to the Random Assignment Problem." Journal of Economic Theory 100, 295-328.

[10] Budish E. (2011) "The Combinatorial Assignment Problem: Approximate Competitive Equilibrium from Equal Incomes," Journal of Political Economy 119, 1061-1103.

[11] Budish. E., Che Y.K., Kojima F. and Milgrom P. (2013). "Designing Random Allocation Mechanisms: Theory and Applications," American Economic Review 103, 585-623.

[12] Carroll, Gabriel (2010) "An Efficiency Theorem for Incompletely Known Preferences." Journal of Economic Theory 145, 2463-2470.

[13] Debreu, Gerard. (1951) "The Coefficient of Resource Utilization" Econometrica, 19(3), 273-292.

[14] Hafalir I. and A. Miralles (2014) "Welfare Maximizing Assignment of Agents to Hierarchical Positions," working paper.

[15] Hara, Chiaki (2005). "Existence of Equilibria in Economies with Bads." Econometrica 73(2), 647-658.

[16] He Y., A. Miralles, and J. Yan. (2012) "Competitive Equilibrium from Equal Incomes in Two-Sided Matching", working paper.

[17] Hylland, A. and R. Zeckhauser (1979) "The Efficient Allocation of Individuals to Positions," Journal of Political Economy 87, 293-314.

[18] Makowski, L., J. M. Ostroy, and U. Segal (1999) "Efficient Incentive Compatible Economies Are Perfectly Competitive," Journal of Economic Theory, 85, 169-225.

[19] Manea, M. (2008) "A Constructive Proof of the Ordinal Efficiency Welfare Theorem." Journal of Economic Theory 141, 276-281.

[20] Manelli, Alejandro M. (1991). "Monotonic Preferences and Core Equivalence." Econometrica 59(1), 123-138

[21] Manjunath, Vikram (2014). "Markets for Fractional Partnerships," working paper. 
[22] Mas-Colell A. (1992) "Equilibrium Theory with Possibly Satiated Preferences," in Equilibrium and Dynamics, Essays in Honor of David Gale, edited by Mukul Majumdar. New York: St. Martins' Press, 201-213.

[23] Mas-Colell A., M. D. Whinston, and J. Green. (1995) Microeconomic Theory. Oxford University Press.

[24] McLennan A. (2002) "Ordinal Efficiency and the Polyhedral Separating Hyperplane Theorem." Journal of Economic Theory 105, 435-449.

[25] Milgrom, Paul R. (2009). "Assignment messages and exchanges." American Economic Journal: Microeconomics, 1(2): 95-113.

[26] Miralles A. (2014) Sequential Pseudomarkets and the Weak Core in Random Assignments, working paper.

[27] Nguyen T., Peivandi A. and Vohra R. (2014) One-Sided Matching with Limited Complementarities, working paper.

[28] Pareto V. (1909). Manuel d'Economie Politique, Paris: M. Giard.

[29] Pycia, M. (2014) The Cost of Ordinality, working paper.

[30] Richter, Michael, and Ariel Rubinstein (2014) "Back to Fundamentals: Equilibrium in Abstract Economies," working paper.

[31] Sonmez, Tayfun, and Utku Unver (2010) "Course Bidding at Business Schools." International Economic Review 51(1), 99-123.

[32] Thomson W. and Zhou L. (1993) "Consistent Allocation Rules in Atomless Economies," Econometrica 61, 575-587. 